\title{
CONICALLY-SIMILAR VISCOUS FLOWS
}

\section{Ross PAULL}

Conically-similar viscous flows constitute the class of steady axissymmetric Navier-Stokes solutions which possess no natural or constructed length scale. This thesis uses basic conservation principles conservation of mass, conservation of ring circulation and conservation of kinematic swirl angular momentum - to postulate that at the origin and on the two half-axes of symmetry there is a total of six independent causes for conically-similar viscous flows. The flows produced by the individual causes are examined in detail and are shown to provide a basic framework for the synthesis of all conically-similar viscous flows.

Two new flows, one with swirl the other swirl-free, are uncovered by this approach. In the swirling example, large kinematic swirl angular momentum production, on a half-axis, leads to a well developed internal boundary layer in the form of a shallow cone, radial jet separating swirlfree and constant swirl circulation flows. In the new swirl-free flow, physical production of ring circulation along the axis of symmetry produces opposed axial jets. When directed inwards, these erupt and discharge into a plane radial jet. Outward directed jets result in induced outer potential flow towards the axis of symmetry.

The flow produced by a point source of axial momentum (Landau [1] and Squire [2]) and the flow associated with a uniform half-line source of mass naturally arise afresh in the classification proposed and complete the basic set of fundamental flows.

In the final part of the thesis, properties and features established

Received 16 March 1983. Thesis submitted to University of Queensland, October 1982. Degree approved March 1983. Supervisor: Professor A.F. Pillow. 
for the basic flow causes aid the investigation of some new conicallysimilar viscous flows within a conical region. Boundary conditions such as no slip or no penetration now lead to non-linear coupling of the fundamental causes. In the first example presented, the surface of a nonporous cone is in constant swirl circulation with a condition of free slip parallel to the generators of the cone. The gradient in the swirl circulation between the cone and the free space half-axis of symmetry rotates and stretches the vortex tubes and leads to production of axial half-plane (ring) circulation and an induced flow towards the cone. The supply of axial momentum to maintain the correct outward flow on the cone then originates from an appropriate point ring circulation cause at the cone apex. A second example exhibiting similar features has a uniform half-line mass source along the axis of a stationary non-porous cone. Nonexistence of solutions now occurs for uniform mass sink strengths greater than $4 \pi \rho v$. The break-down is caused by an infinite strength inward axial jet which develops to account for the cone-generated ring circulation accumulated about the axis of symmetry by the uniform mass sink there.

In the final problem, the cone in the second example is specialized to a plane and a uniform half-line source of kinematic swirl angular momentum is superimposed on the uniform mass source. Sufficiently large swirl circulations produce unbounded flows because an infinite strength axial jet develops from the ring circulation which is generated by the severe rotation and stretching of vortex tubes in the boundary layer about the axis of symmetry. This outward jet could equally well be produced by an equivalent distribution of ring circulation causes along the axis of symmetry. The domain of existence for the swirling flow and the equivalent swirl-free flow are then the same.

Mathematically speaking, the absence of a length scale in all conically-similar viscous flows means that the radial dependence of physical quantities can be determined by dimensional analysis. The governing partial differential equations corresponding to the basic conservation principles then reduce to a sixth order non-autonomous system of two nonlinear coupled ordinary differential equations. The motion naturally divides into its swirling and swirl-free parts. In constant swirl circulation flows with no physical boundary, the strengths of the swirl-free fundamental causes on the half-axes provide three boundary 
conditions which allow the fourth order differential equation governing the axial half-plane flow to be integrated into the form of a Riccati equation. A non-linear integral condition specifying the strength of a point ring circulation cause at the origin determines the constant of integration. When there is non-constant swirl circulation, the second order ordinary differential equation controlling the distribution of swirl is no longer trivial and non-linear coupling with the axial half-plane flow generalizes the Riccati equation to an integro-differential equation.

Closed-form solutions to this system of equations exist only for constant swirl circulation flows. In all cases, however, the asymptotic forms of the solution for small and large strengths provide an efficient means of exploring flow behaviour. Small strengths result in (almost) Stokes flow. The solution for the streamfunction can then be expressed as a convergent series in powers of the small strength. The qualification almost is necessary since for even the smallest non-zero strength there may exist narrow regions of non-uniformity about the axis of symmetry where convection (of, say, ring circulation) is not everywhere subdominant to diffusion. Large strengths result in one of two possibilities. If the axial half-plane flow acts to oppose diffusion of ring circulation from all sources of ring circulation, a boundary layer develops about these causes. The first order concentration of ring circulation away from such a layer is then exponentially small and an induced outer potential flow results. Convection aiding diffusion of ring circulation from a source of ring circulation, however, results in a distribution of ring circulation throughout the flow field.

These three limiting forms of the solution also provide valuable estimates for determining flow properties. In particular, since solutions for general strengths are clamped between these extremes, domains for existence can be determined. Differential inequality techniques can then be used to establish uniqueness, to investigate the distribution of ring circulation and to examine existence of flow separatrices.

In all problems studied, microcomputer generated solutions and plotted figures illustrate and support the analytic and asymptotic results. A convenient representation of flow trends and behaviour is contained in the axial half-plane and isometric views of the flow streamlines. 


\section{References}

[1] L. Landau, "A new exact solution of the Navier Stokes equation", C.R. (Doklady) Acad. Sci. URSS (N.S.) 43 (1944), 286-288.

[2] H.B. Squire, "The round laminar jet", Quart. J. Mech. Appl. Math. 4 (1951), 321-329.

Department of Mathematics,

University of Queensland,

St Lucia,

Queensland 4067,

Australia. 